\title{
Unmet Needs in Japanese Patients Who Report Insufficient Efficacy with Triptans for Acute Treatment of Migraine: Retrospective Analysis of Real- World Data
}

\author{
Koichi Hirata $\cdot$ Kaname Ueda (1) $\cdot$ Mika Komori $\cdot$ Wenyu Ye \\ Yongin Kim $\cdot$ Sarah Cotton $\cdot$ James Jackson $\cdot$ Tamas Treuer
}

Received: September 28, 2020 / Accepted: November 19, 2020 / Published online: December 11, 2020

(C) The Author(s) 2020

\begin{abstract}
Introduction: Migraine attacks notably impact people's daily lives, health-related quality of life (HRQoL), and ability to work. Triptans are widely used as acute medication for a migraine attack but are ineffective, poorly tolerated, or contraindicated in some patients. HRQoL and work productivity are therefore likely to pose particular problems for patients whose migraine attacks do not respond sufficiently to triptan acute treatment. This real-world study aimed to determine whether migraine-related HRQoL, disability, and work productivity differed between triptan insufficient responders (TIRs)
\end{abstract}

Electronic supplementary material The online version of this article (https://doi.org/10.1007/s40122020-00223-y) contains supplementary material, which is available to authorized users.

K. Hirata

Department of Neurology, Dokkyo Medical

University, Tochigi, Japan

K. Ueda $(\varangle) \cdot$ M. Komori

Eli Lilly Japan K.K, Kobe, Japan

e-mail: ueda_kaname@lilly.com

W. Ye $\cdot$ Y. Kim

Eli Lilly and Company, Indianapolis, IN, USA

S. Cotton · J. Jackson

Adelphi Real World, Bollington, UK

T. Treuer

Lilly Hungária Kft, Budapest, Hungary and sufficient responders (TSRs) receiving this acute treatment for migraine in Japan.

Methods: This was a retrospective analysis of 2017 Adelphi Migraine Disease Specific Programme cross-sectional survey data collected from physicians and their consulting patients with migraine in Japan. Patients had to be receiving a triptan as their sole acute prescribed medication for migraine. TIRs were defined as patients who achieved headache pain freedom within $2 \mathrm{~h}$ of taking triptan acute treatment in no more than three of five migraine attacks. Differences in outcomes between TIRs and TSRs were examined in adjusted analyses using a multivariable general linear model.

Results: Of 200 patients receiving a triptan as their sole prescribed acute treatment for migraine, $88(44.0 \%)$ were classed as TIRs. Migraine-Specific Quality of Life Questionnaire scores were significantly lower-indicating poorer HRQoL-among TIRs than TSRs, as were mean EuroQol 5-dimension utility and visual analog scale scores $(p<0.05$ for comparisons). TIRs also reported significantly $(p \leq 0.003)$ greater impairment than TSRs across all Work Productivity and Activity Impairment domains, with the exception of work time missed. Migraine disability was higher among TIRs than TSRs.

Conclusion: Migraine attacks had a negative impact on the HRQoL, disability, and work productivity of people with migraine in Japan reporting insufficient efficacy with acute triptan 
treatment, highlighting the need for more effective acute treatment options.

Keywords: Adelphi Migraine Disease Specific Programme; Health-related quality of life; Japan; Migraine; Real-world; Retrospective study; Triptan; Work productivity

\section{Key Summary Points}

Why carry out this study?

Migraine attacks have a notable impact on people's daily lives, health-related quality of life (HRQoL), and ability to work.

HRQoL and work productivity are likely to pose particular problems for patients whose migraine attacks do not respond sufficiently to commonly used acute treatments such as triptans.

This real-world study aimed to determine whether migraine-related HRQoL, disability, and work productivity differed between people reporting insufficient efficacy (triptan insufficient responders) and sufficient efficacy (triptan sufficient responders) with triptans as acute treatment for migraine in Japan.

\section{What was learned from the study?}

Migraine-related disability was higher, and migraine-related HRQoL and work productivity were significantly lower, in triptan insufficient responders (based on insufficient efficacy) than in triptan sufficient responders.

The negative impact of migraine attacks on the HRQoL, ability to perform in daily life, and work productivity of people in Japan reporting insufficient efficacy with acute triptan treatment highlights the need for additional effective acute treatment options.

\section{DIGITAL FEATURES}

This article is published with digital features, including a summary slide, to facilitate understanding of the article. To view digital features for this article go to https://doi.org/10.6084/ m9.figshare.13251809.

\section{INTRODUCTION}

Migraine is highly prevalent worldwide and places a considerable burden on patients [1]. In 2016 , the prevalence of migraine in Japan was reported to be 17.8 (95\% uncertainty interval 17.1-18.5) million, accounting for 0.75 (0.48-1.05) million years lived with disability (YLD) [2]. Migraine was the fourth leading cause of YLD [3].

Pharmacological management options for migraine include acute treatment to relieve pain and its associated symptoms during an attack or to limit the duration and severity of an attack, emergency treatment, and preventive treatment [4]. There is, however, evidence that current treatment approaches are not sufficiently meeting the needs of people with migraine. A US longitudinal population-based study (American Migraine Prevalence and Prevention) found that, of the more than 5500 people with episodic migraine, $>40 \%$ reported having at least one unmet treatment need with their current acute treatment, among whom 37.4\% were dissatisfied with their acute treatment regimen [5]. A recent systematic review highlighted high levels of unmet needs, relating to a lack of awareness and under-diagnosis of migraine, and a need for better management and therapies for treatment, among patients with the disease in East Asia, including Japan [6]. In Japan, an increasing amount of realworld data suggests that migraine (both episodic and chronic) poses a notable disease burden and highlights high levels of unmet need with prescribed acute and preventive medication among people with migraine $[7,8]$.

In Japan, triptans are among the medications recommended by the Japanese Society of Neurology and the Japanese Headache Society for the acute treatment of a migraine attack [9]. 
Globally, triptans are commonly used for this purpose [10] but are not effective in all patients, with rates of pain freedom at $2 \mathrm{~h}$ post-dose (the recommended primary endpoint for assessing the efficacy of such treatments [11]) ranging from 18 to $50 \%$ [10]. Additionally, some individuals have contraindications to triptans (e.g., cardiovascular/cerebrovascular conditions such as ischemic heart disease, stroke, transient ischemic attack, and uncontrolled hypertension [12]) or are intolerant of or unwilling to take these agents [13-15]. Patient persistence with triptan therapy is low, with the 1-year probability of discontinuation among all triptan users reported to be $30-60 \%$ [16, 17]. Lack of acute medication efficacy has been linked with an increased risk of progression from episodic to chronic migraine [18].

People with migraine experience attacks of intense pain and other symptoms, such as nausea, vomiting, photophobia, and phonophobia, which can notably impact upon their daily life, health-related quality of life (HRQoL), and ability to work $[19,20]$. Reduced HRQoL and work productivity are therefore likely to pose particular problems to patients whose migraine attacks do not respond sufficiently to triptans. It is important to realize the burden of insufficient response on patients with migraine and the need for more effective acute treatment options. To the best of our knowledge, no studies have examined outcomes in this patient population who do not respond to triptan treatment in Japan.

The main aim of this retrospective analysis of 2017 Japan Adelphi Migraine Disease Specific Programme (DSP) cross-sectional survey data was to determine whether migraine-related HRQoL, disability, and work productivity differed between people reporting insufficient efficacy (termed here triptan insufficient responders [TIRs]) or sufficient efficacy (triptan sufficient responders [TSRs]) with prior triptan acute therapy for migraine in Japan. We also aimed to describe and identify characteristics and factors associated with insufficient triptan efficacy.

\section{METHODS}

\section{Study Design}

This was a retrospective analysis of real-world, point-in-time, cross-sectional survey data on migraine treatment practice, patient demographics, clinical features, outcomes, migrainerelated HRQoL, disability, and work productivity collected from physicians and their consulting patients with migraine as part of the Japan Adelphi Migraine DSP over the period October 2017-March 2018. The DSP is Adelphi Real World's proprietary, real-world evidence generation program.

Full details of the standardized methodology used in the DSPs [21] and of the methodological details of the Japan Adelphi Migraine DSP data have been published $[7,8]$. Briefly, physicians completed a patient record form (PRF) for nine consecutive adult patients (aged $\geq 18$ years) with migraine, collecting information on the patient's demographics and clinical characteristics, including headache diagnoses (e.g., medication overuse, tension-type, chronic, episodic), average migraine severity over the past 3 months, average monthly attack frequency over the past 3 months, symptoms, and prescribed medication; concomitant diagnoses; and treatment history. To achieve an oversampling of patients for whom at least one prior preventive treatment had failed, every tenth patient needed to meet this requirement and may not have been consecutive.

The physician then invited each patient for whom a PRF had been completed to fill in a confidential patient self-completion (PSC) form, which included questions about the patient's demographics, current symptoms, and therapeutic response to and level of satisfaction with acute prescribed medication (response options ranged from extremely satisfied to extremely dissatisfied). Patients also provided information on additional patient-reported outcomes, such as the impact of migraine on their HRQoL (using the Migraine-Specific Quality of Life questionnaire [MSQ] version 2.1 [22] and the EuroQol 5-dimension 5-level questionnaire [EQ5D-5L] [23]), their headache-related disability 
over the past 3 months (using the Migraine Disability Assessment [MIDAS] test [24, 25]), and their work productivity and activity impairment due to migraine (using the Work Productivity and Activity Impairment questionnaire: Migraine [WPAI:Migraine] [26]).

Patients provided informed consent for use of their anonymized and aggregated data for research and publication in scientific journals by means of a check box on the front page of the PSC. Data were collected in such a way that patients and physicians could not be identified directly. All data were aggregated and de-identified before receipt by Adelphi Real World. The DSP methodology and questionnaires were reviewed by an official independent body, Freiburger Ethik-Kommission International (FEKI), which granted ethical approval. As this was a retrospective analysis that used de-identified data that had been previously collected, patient participants were not required to provide formal Consent to Release Information forms for the current analyses; the original consent from those who provided data in the DSP covered the planned analyses in this study. The study was conducted in accordance with the applicable regulations of Japan (the Ethical Guidelines for Medical and Health Research Involving Human Subjects).

\section{Study Population}

\section{Physicians}

Physician participants in the Japan Adelphi Migraine DSP were either internists (primary care physicians or general practitioners) or neurologists currently treating patients with migraine. Participating internists and neurologists were required to make treatment decisions for six or more people with migraine in a typical month.

\section{Patients}

Patient participants in the Japan Adelphi Migraine DSP were adults with a physicianconfirmed diagnosis of migraine actively seeking care from their physician. To be included in the current analysis, patients had to be currently receiving any formulation of a triptan as their sole acute prescribed medication for migraine, to have completed a PSC, and to have answered the following survey question, considering their condition over the past 3 months: "In approximately how many migraine attacks would you say your prescribed acute medication stops the pain entirely within 2 hours of taking the medication?" In response, patients could select from none, one, two, three, four, or five out of five attacks.

On the basis of their responses to the above question, patient participants were assigned to one of two cohorts: TIRs or TSRs. TIRs were defined as patients who, after taking a triptan as an acute treatment, achieved headache pain freedom within $2 \mathrm{~h}$ in no more than three of five migraine attacks, whereas TSRs were defined as those who, after taking a triptan as an acute treatment, achieved headache pain freedom within $2 \mathrm{~h}$ in at least four of five migraine attacks [28, 29].

\section{Study Endpoints}

The primary endpoint of the study was to determine whether migraine-associated HRQoL, disability, and work productivity differed significantly between TIRs and TSRs. Secondary endpoints included the identification of characteristics and factors associated with insufficient triptan efficacy.

\section{Outcomes}

Migraine-associated HRQoL, disability, and work productivity were assessed using validated Japanese translations of the MSQ (version 2.1), EQ-5D-5L, MIDAS questionnaire, and WPAI:Migraine questionnaire.

The MSQ (version 2.1) is a migraine-specific instrument that allows patients to rate how migraine attacks restrict three aspects of their HRQoL: their daily social and work-related activities (Role Function - Restrictive; seven items), how migraine attacks prevent these activities (Role Function - Preventive; four items), and the emotional impact of migraine attacks (Emotional Function; three items), using a scale of 1 (none of the time) to 6 (all of the time) [22]. Computed raw scores for the MSQ total score, and each of the three domains, were 
linearly transformed to a $0-100$ scale (100 indicates full functionality).

The EQ-5D-5L comprises the EQ-5D-5L descriptive system (measuring mobility, selfcare, usual activities, pain/discomfort, and anxiety/depression) and the EQ-5D visual analog scale (VAS) [23]. The EQ-5D-5L utility score provided a numeric index of the health state of the patient population using Japanese tariffs (higher scores indicate better health). The EQ$5 \mathrm{D}$ VAS score is the average numeric rating of the VAS score $(0=$ worst imaginable health state; 100 = best imaginable health state).

For the MIDAS questionnaire, patients answer five questions recording the number of days reported as missing work or with reduced productivity at work or home and missed social events due to a headache over the past 3 months [24, 25, 27]. Higher total scores (total number of days summed from the answers to the five questions) indicate greater disability $(0-5=$ little/no disability, $6-10=$ mild disability, $11-20=$ moderate disability, $\geq 21=$ severe disability).

The WPAI:Migraine questionnaire calculates four scores for percentage work time missed (absenteeism), percentage impairment while working (presenteeism), percentage overall work impairment, and percentage activity impairment [26], with each score ranging from 0 to $100 \%$ after transformation (higher scores indicate greater impairment).

Additionally, physicians assessed each patient's severity of migraine over the past 3 months using a 10-point Likert scale $(1=$ very mild; 10 = very severe). "Do not know" was also a response option.

\section{Statistical Analysis}

To compare differences in the baseline characteristics and outcomes between TIRs and TSRs, continuous measures were assessed using twosample $t$ tests, and categorical measures were assessed using the chi-square test or Fisher's exact test if the expected count was $<5$.

To control for potential confounding effects on the outcomes of migraine-associated HRQoL, disability, and work productivity between TIRs and TSRs, an adjusted analysis using a multivariable general linear model (GLM) was conducted to examine differences between the two groups. The GLM was fitted using TIRs versus TSRs as a main effect and controlled for age, sex, migraine headache day frequency, comorbidities, duration of illness, preventive medication use, and presence of aura. Least squares (LS) means with 95\% confidence intervals (CIs) from the GLM analysis were reported for each group.

Factors associated with insufficient triptan efficacy (vs. sufficient triptan efficacy) were identified using logistic regression with backward variable selection. Candidate continuous covariates included age, MIDAS total score, EQ5D-5L utility score, physician-reported monthly headache days over the past 3 months, and physician-reported headache severity over the past 3 months. Candidate categorical covariates included physician specialty, patient sex, diagnosis of migraine with aura, diagnosis of migraine without aura, diagnosis of tensiontype headache, comorbidities (depression, anxiety, neck pain), diagnosis of menstrual migraine or menstrual-related migraine, symptoms currently experienced (nausea, vomiting), when acute medication is taken relative to the start of the attack ("at the first sign of a migraine" [before the pain starts], "when the pain starts," or "after the pain has started and I have an idea of how severe it is"), taking overthe-counter (OTC) medication for migraine attacks, and whether the patient ever received preventive medication for their migraine. A significance level of 0.1 was required for a variable to stay in the model. Age and sex were forced to remain in the model. Odds ratios (ORs), 95\% CIs, and $p$ values from the final logistic regression model were reported.

Summary statistical information was based on non-missing data. No imputation strategy was implemented for missing data. The number of patients excluded because of missingness from adjusted analyses of the GLM model included 24 patients for MIDAS total score, three patients for EQ-5D-5L utility score and MSQ total score, and 87 patients for WPAI. For the backward logistic regression model, three patients $(3 / 200=1.5 \%)$ were excluded because 
of missingness in covariates. All statistical tests were conducted at a two-sided 5\% significance level without any multiplicity adjustment. Analyses were conducted using SAS Enterprise Guide 7.15 (SAS Institute, Cary, NC, USA).

\section{RESULTS}

The study population comprised 200 patients who were receiving a triptan as their sole prescribed acute treatment for migraine at the time of the survey, had completed a PSC, and had provided information on the efficacy of their triptan therapy over five migraine attacks in their PSC form. Of these patients, 88 (44.0\%) were classified as TIRs and $112(56.0 \%)$ were classified as TSRs (Fig. 1).

There were no significant differences in demographics between TIRs and TSRs. TIRs were less likely to be managed by an internist (52.3 vs. $66.1 \%)$ and more likely to be managed by a neurologist (47.7 vs. $33.9 \%)$ than TSRs at the time of the current consultation $(p=0.048)$ (Table 1). TIRs were significantly more likely than TSRs to have a diagnosis of migraine without aura (62.5 vs. $42.0 \%, p=0.004)$ or chronic migraine $(12.5$ vs. $0.9 \%, p<0.001)$ and significantly less likely to have a diagnosis of migraine with aura (35.2 vs. $55.4 \%, p=0.005)$ (Table 1). Similar levels of other headache diagnoses (tension-type, rebound/medication overuse, and menstrual/menstrual-related headache) were seen between groups.

Neck pain was the most common concomitant condition experienced by all patients (13.0\%) (Table 2). No significant differences in diagnosed concomitant conditions, including the number of conditions or the incidence of cardiovascular conditions, were reported by physicians between TIRs and TSRs. Current cardiovascular risk was assessed by the physician as mild or moderate in similar proportions of TIRs and TSRs.

Currently experienced migraine-related symptoms were generally reported in similar proportions of TIRs and TSRs, although sensitivity to sound (42.0 vs. $27.7 \%$ ), pain worsened by activity/movement (40.9 vs. $25.9 \%)$, and muscle weakness/fatigue (17.0 vs. $6.3 \%)$ were

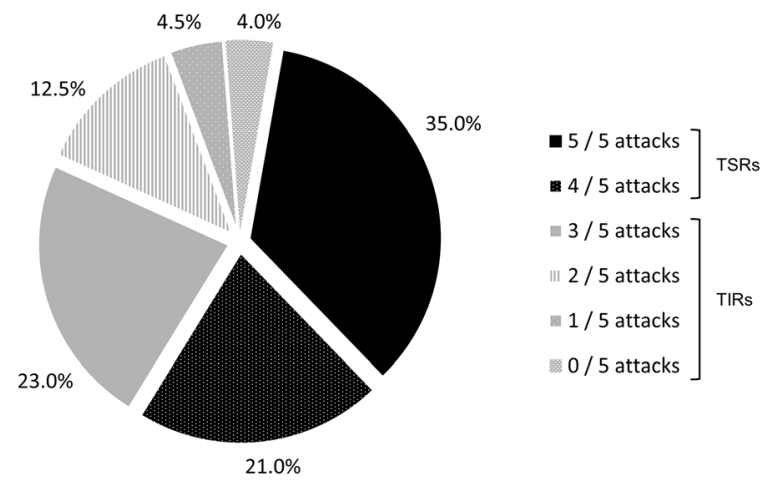

Fig. 1 Derivation of triptan insufficient responder (TIR) and triptan sufficient responder (TSR) cohorts $(N=200)$, based on response to the question: "In approximately how many migraine attacks would you say your prescribed acute medication stops the pain entirely within $2 \mathrm{~h}$ of taking the medication?" TIRs were defined as patients who, after taking a triptan as an acute treatment, achieved headache pain freedom within $2 \mathrm{~h}$ in no more than three of five migraine attacks, whereas TSRs were defined as those who, after taking a triptan as an acute treatment, achieved headache pain freedom within $2 \mathrm{~h}$ in at least four of five migraine attacks

reported significantly more commonly by TIRs than by TSRs (all $p<0.05$ ) (Table S1 in the electronic supplementary material). Physicians reported that, over the 3 months prior to participation in the study, TIRs experienced significantly more monthly headache days (mean 5.7 vs. 4.5 , respectively; $p=0.023$ ) and monthly migraine headache days (4.8 vs. 3.7, respectively; $p=0.013$ ) than TSRs. Migraine over the past 3 months was significantly more severe in TIRs than in TSRs (scored as 6.4 vs. 5.2, $p<0.001$ ) (Table 3).

The most common currently prescribed triptans were sumatriptan (for $38.5 \%$ of patients) and zolmitriptan (22.0\%); physicians reported that TIRs were more likely to have tried a larger number of unique triptans than TSRs, although the difference was not statistically significant (Table 3). TIRs were significantly more likely than TSRs to have ever used (53.4 vs. $33.9 \%, p=0.006)$ or to be currently receiving (52.3 vs. $32.1, p=0.004)$ prescribed preventive treatment (Table 3). Patient-reported data indicated that timing of triptan administration differed significantly ( $p=0.033$ ) between TIRs and 
Table 1 Patient characteristics

\begin{tabular}{|c|c|c|c|c|}
\hline Characteristic & Total $(N=200)$ & TIRs $(n=88)$ & TSRs $(n=112)$ & $p$ value \\
\hline \multicolumn{5}{|l|}{ PSC data } \\
\hline Age in years, mean $(S D)$ & $43.7(13.1)$ & $43.7(12.4)$ & $43.7(13.7)$ & $0.980^{\mathrm{a}}$ \\
\hline Female, $n(\%)$ & $153(76.5)$ & $71(80.7)$ & $82(73.2)$ & $0.216^{\mathrm{b}}$ \\
\hline Married, $n(\%)$ & $131(65.5)$ & $52(59.1)$ & $79(70.5)$ & $0.153^{\mathrm{c}}$ \\
\hline Smoking status: current or prior smoker, $n(\%)$ & $48(24.6)$ & $22(25.8)$ & $26(23.6)$ & $0.476^{\mathrm{b}}$ \\
\hline Employed, $n(\%)$ & $142(72.4)$ & $64(73.6)$ & $78(71.6)$ & $0.755^{\mathrm{b}}$ \\
\hline \multicolumn{5}{|l|}{ PRF data } \\
\hline Specialty of consulting physician, $n(\%)$ & & & & $0.048^{b}$ \\
\hline Internist $^{\mathrm{d}}$ & $120(60.0)$ & $46(52.3)$ & $74(66.1)$ & \\
\hline Neurologist & $80(40.0)$ & $42(47.7)$ & $38(33.9)$ & \\
\hline \multicolumn{5}{|l|}{ Headache diagnosis, $n(\%)$} \\
\hline Migraine with aura & $93(46.5)$ & $31(35.2)$ & $62(55.4)$ & $0.005^{\mathrm{b}}$ \\
\hline Migraine without aura & $102(51.0)$ & $55(62.5)$ & $47(42.0)$ & $0.004^{b}$ \\
\hline Menstrual/menstrual-related migraine & $33(16.5)$ & $16(18.2)$ & $17(15.2)$ & $0.570^{\mathrm{b}}$ \\
\hline Chronic migraine $^{e}$ & $12(6.0)$ & $11(12.5)$ & $1(0.9)$ & $<0.001^{\mathrm{b}}$ \\
\hline Rebound/medication overuse headache & $4(2.0)$ & $3(3.4)$ & $1(0.9)$ & $0.322^{\mathrm{c}}$ \\
\hline Tension-type headache & $52(26.0)$ & $26(29.5)$ & $26(23.2)$ & $0.311^{\mathrm{b}}$ \\
\hline
\end{tabular}

Percentages are calculated as proportion of non-missing data

Bold text indicates statistical significance for TIRs versus TSR

PRF patient record form (completed by the physician), PSC patient self-completion, SD standard deviation, TIR triptan insufficient responders (based on efficacy), TSR triptan sufficient responders (based on efficacy)

a $t$ test

b Chi-square test

c Fisher's exact test

d Primary care physician/general practitioner

e Defined as $\geq 15$ headache days per month for $>3$ months in the past 12 months

TSRs, with TIRs more likely than TSRs to take triptan acute medication when/after the pain starts ( 55.2 vs. $49.1 \% / 13.8$ vs. $5.4 \%)$ than at the first sign of pain (31.0 vs. $45.5 \%)$. TIRs were also significantly more likely to need to take extra doses to relieve their pain (64.8 vs. $34.3 \%)$ and to be taking OTC medications (19.5 vs. $4.5 \%)$ (both $p<0.001$ ) (Table 4). Patient-reported satisfaction with prescribed triptan acute medication differed significantly $(p<0.001)$ between TIRs and TSRs, with greater satisfaction levels being more common for TSRs and lower satisfaction levels or dissatisfaction being more common for TIRs (Table 4).

Figures 2, 3 show the estimated LS means with 95\% CIs for the reported outcomes in each group obtained from the adjusted GLM. In adjusted analyses, HRQoL was reported to be poorer among TIRs than among TSRs, as shown by lower MSQ total (71.0 [95\% CI 67.1, 74.8] vs. 77.5 [95\% CI 74.1, 81.0]; $p=0.009$ ), Role Function - Restrictive (68.5 [95\% CI 64.6, 72.4] 
Table 2 Concomitant conditions in TIRs and TSRs (PRF data)

\begin{tabular}{lcccc}
\hline Concomitant conditions, $\boldsymbol{n}(\%)$ & Total $(\boldsymbol{N}=\mathbf{2 0 0})$ & TIRs $(\boldsymbol{n}=\mathbf{8 8})$ & TSRs $(\boldsymbol{n}=\mathbf{1 1 2})$ & $\boldsymbol{p}$ value \\
\hline Neck pain & $26(13.0)$ & $13(14.8)$ & $13(11.6)$ & $0.509^{\mathrm{a}}$ \\
Anxiety & $9(4.5)$ & $5(5.7)$ & $4(3.6)$ & $0.511^{\mathrm{b}}$ \\
Stress & $13(6.5)$ & $5(5.7)$ & $8(7.1)$ & $0.677^{\mathrm{a}}$ \\
Sleep disorders & $8(4.0)$ & $3(3.4)$ & $5(4.5)$ & $1.000^{\mathrm{b}}$ \\
Depression & $5(2.5)$ & $3(3.4)$ & $2(1.8)$ & $0.656^{\mathrm{b}}$ \\
Asthma/allergic rhinitis & $9(4.5)$ & $4(4.5)$ & $5(4.5)$ & $1.000^{\mathrm{b}}$ \\
Back pain & $4(2.0)$ & $2(2.3)$ & $2(1.8)$ & $1.000^{\mathrm{b}}$ \\
Thyroid disease & $1(0.5)$ & 0 & $1(0.9)$ & $1.000^{\mathrm{b}}$ \\
No. of diagnosed concomitant conditions, mean (SD) & $0.59(1.0)$ & $0.56(0.8)$ & $0.62(1.1)$ & $0.664^{\mathrm{c}}$ \\
Currently has a cardiovascular condition, $\mathrm{n}(\%)$ & $27(13.5)$ & $12(13.6)$ & $15(13.4)$ & $0.960^{\mathrm{a}}$ \\
Hypertension & $25(12.5)$ & $10(11.4)$ & $15(13.4)$ & $0.667^{\mathrm{a}}$ \\
Angina & $1(0.5)$ & 0 & $1(0.9)$ & $1.000^{\mathrm{b}}$ \\
Other ${ }^{\mathrm{b}}$ & $3(1.5)$ & $2(2.3)$ & $1(0.9)$ & - \\
Current cardiovascular risk, ${ }^{\mathrm{b}} n$ (\%) & & & & $0.821^{\mathrm{a}}$ \\
Mild & $169(86.7)$ & $74(86.0)$ & $95(87.2)$ & $14(12.8)$ \\
Moderate & $26(13.3)$ & $12(14.0)$ & \\
\hline
\end{tabular}

Percentages are calculated as proportion of non-missing data

PRF patient record form (completed by the physician), $S D$ standard deviation, TIR triptan insufficient responders (based on efficacy), TSR triptan sufficient responders (based on efficacy)

a Chi-square test for TSRs versus TIRs

b Fisher's exact test for TSRs versus TIRs

${ }^{c} t$ test for TSRs versus TIRs

${ }^{d}$ No patient in either group had cerebrovascular disease, ischemic heart disease, congestive heart failure, hypotension, atrial fibrillation, arrhythmia, deep vein thrombosis, cardiomyopathy, coronary artery disease, peripheral vascular disease, mitral valve disease, or Raynaud's disease

${ }^{\mathrm{e}}$ Subjective opinion of the physician; no patient was assessed as being at severe risk

vs. 74.7 [95\% CI 71.2, 78.2]; $p=0.015)$, Role Function - Preventive (75.9 [95\% CI 71.6, 80.2] vs. 81.4 [95\% CI 77.6, 85.2]; $p=0.043)$, and Emotional Function (70.3 [95\% CI 66.0, 74.6] vs. 78.9 [95\% CI 75.0, 82.7]; $p=0.002$ ) scores (Fig. 2a) and significantly lower mean EQ-5D-5L utility $(0.80$ [95\% CI 0.76, 0.84] vs. 0.88 [95\% CI $0.85,0.92] ; p<0.001)$ and EQ-5D VAS scores (68.8 [95\% CI 65.1, 72.4] vs. 80.7 [95\% CI 77.4, 83.9]; $p<0.001$; Fig. 2b). Migraine-related disability was also reported to be greater among TIRs than among TSRs as shown by a (non- significantly) higher MIDAS total score (18.8 [95\% CI 12.3, 25.2] vs. 11.5 [95\% CI 5.5, 17.6]; $p=0.087$; Fig. 2c).

TIRs also reported significantly greater impairment and less productivity than TSRs in all WPAI domains (impairment while working: $57.2 \%$ [95\% CI 47.7, 66.7] vs. 37.0\% [95\% CI 28.6, 45.4]; $p=0.001$; overall work impairment: $57.9 \%$ [95\% CI $48.3,67.5]$ vs. $37.7 \%$ [95\% CI 29.1, 46.2]; $p=0.002$; activity impairment: $56.6 \%$ [95\% CI $49.4,63.9]$ vs. $42.7 \%$ [95\% CI $36.3,49.2] ; p=0.003)$, with the exception of 
Table 3 Migraine characteristics and treatment (PRF data)

\begin{tabular}{|c|c|c|c|c|}
\hline Characteristic and treatment & $\begin{array}{l}\text { Total } \\
(N=200)\end{array}$ & $\begin{array}{l}\text { TIRs } \\
(n=88)\end{array}$ & $\begin{array}{l}\text { TSRs } \\
(n=112)\end{array}$ & $p$ value \\
\hline Monthly headache days over past 3 months, mean (SD) & $5.0(3.5)$ & $5.7(4.1)$ & $4.5(2.9)$ & $0.023^{\mathrm{a}}$ \\
\hline $\begin{array}{l}\text { Monthly migraine headache days over past } 3 \text { months, mean } \\
\text { (SD) }\end{array}$ & $4.2(3.1)$ & $4.8(3.6)$ & $3.7(2.5)$ & $0.013^{\mathrm{a}}$ \\
\hline Severity of migraine over past 3 months $^{\mathrm{b}}$ & $5.7(2.0)$ & $6.4(1.8)$ & $5.2(2.0)$ & $<0.001^{\mathrm{a}}$ \\
\hline \multicolumn{5}{|l|}{ Currently prescribed triptans, $n(\%)$} \\
\hline Sumatriptan & $77(38.5)$ & $37(42.0)$ & $40(35.7)$ & $0.361^{\mathrm{c}}$ \\
\hline Zolmitriptan & $44(22.0)$ & $15(17.0)$ & $29(25.9)$ & $0.134^{\mathrm{c}}$ \\
\hline Naratriptan & $31(15.5)$ & $15(17.0)$ & $16(14.3)$ & $0.592^{c}$ \\
\hline Rizatriptan & $30(15.0)$ & $11(12.5)$ & $19(17.0)$ & $0.380^{\mathrm{c}}$ \\
\hline Eletriptan & $19(9.5)$ & $11(12.5)$ & $8(7.1)$ & $0.200^{c}$ \\
\hline No. of ever used unique triptans, $n$ (\%) & & & & $0.068^{\mathrm{d}}$ \\
\hline 1 & $174(87.0)$ & $74(84.1)$ & $100(89.3)$ & \\
\hline 2 & $22(11.0)$ & $10(11.4)$ & $12(10.7)$ & \\
\hline 3 & $4(2.0)$ & $4(4.5)$ & 0 & \\
\hline Ever used preventive medication, $n$ (\%) & $85(42.5)$ & $47(53.4)$ & $38(33.9)$ & $0.006^{\mathrm{c}}$ \\
\hline Currently prescribed preventive medication, $n$ (\%) & $82(41.0)$ & $46(52.3)$ & $36(32.1)$ & $0.004^{c}$ \\
\hline
\end{tabular}

Percentages are calculated as proportion of non-missing data Bold text indicates statistical significance for TSRs versus TIRs

$P R F$ patient record form (completed by the physician), $S D$ standard deviation, TIR triptan insufficient responders (based on efficacy), TSR triptan sufficient responders (based on efficacy)

${ }^{\mathrm{a}} t$ test

${ }^{\mathrm{b}}$ Reported as average severity over the past 3 months using a scale from 1 (very mild) to 10 (very severe)

c Chi-square test

d Fisher's exact test

work time missed $(6.4[95 \%$ CI $2.4,10.4]$ vs. 2.6 $[95 \% \mathrm{CI}-1.0,6.2] ; p=0.145)$, for which the difference did not achieve statistical significance (Fig. 3).

These findings were also apparent in unadjusted analyses, with the exception of a significantly higher MIDAS score in TIRs than in TSRs (18.4 vs. $6.5, p=0.01$ ), which was reflected in the patient distribution across all disability categories (Table S2 in the electronic supplementary material).

\section{Characteristics Associated with Insufficient Triptan Efficacy}

Factors associated with insufficient triptan efficacy, as identified by backward logistic regression analysis, are shown in Table 5 . The odds of being a TIR were higher for patients taking OTC medications for their migraine than for those not taking OTC medications (OR 4.679 [95\% CI $1.524,14.371] ; p=0.007$ ), for those taking their prescribed medication after the pain had started rather than at the first sign of a migraine attack 
Table 4 Triptan acute treatment patterns and patient satisfaction with prescribed triptan acute medication in TIRs and TSRs (PSC data)

\begin{tabular}{|c|c|c|c|c|}
\hline Factor, $n(\%)$ & $\begin{array}{l}\text { Total } \\
(N=200)\end{array}$ & $\begin{array}{l}\text { TIRs } \\
(n=88)\end{array}$ & $\begin{array}{l}\text { TSRs } \\
(n=112)\end{array}$ & $p$ value \\
\hline \multicolumn{4}{|l|}{ Time of administration of triptan acute medication } & $\mathbf{0 . 0 3 3 ^ { \mathrm { a } }}$ \\
\hline At first sign of a migraine & $78(39.2)$ & $27(31.0)$ & $51(45.5)$ & \\
\hline When the pain starts & $103(51.8)$ & $48(55.2)$ & $55(49.1)$ & \\
\hline After the pain has started & $18(9.0)$ & $12(13.8)$ & $6(5.4)$ & \\
\hline \multicolumn{4}{|l|}{ Overall satisfaction with prescribed triptan acute medication } & $<0.001^{\mathrm{b}}$ \\
\hline Extremely satisfied & $41(20.7)$ & $4(4.5)$ & $37(33.6)$ & \\
\hline Satisfied & 88 (44.4) & $30(34.1)$ & $58(52.7)$ & \\
\hline Slightly satisfied & $47(23.7)$ & $38(43.2)$ & $9(8.2)$ & \\
\hline Neither satisfied nor dissatisfied & $11(5.6)$ & $7(8.0)$ & $4(3.6)$ & \\
\hline Slightly dissatisfied & $5(2.5)$ & $4(4.5)$ & $1(0.9)$ & \\
\hline Dissatisfied & $5(2.5)$ & $5(5.7)$ & 0 & \\
\hline Extremely dissatisfied & $1(0.5)$ & 0 & $1(0.9)$ & \\
\hline $\begin{array}{l}\text { Need to take extra dose(s) of triptan acute medication to } \\
\text { relieve pain }\end{array}$ & $94(48.0)$ & $57(64.8)$ & $37(34.3)$ & $<0.001^{\mathrm{a}}$ \\
\hline Currently taking OTC medications for migraine & $22(11.1)$ & $17(19.5)$ & $5(4.5)$ & $<0.001^{\mathrm{a}}$ \\
\hline \multicolumn{5}{|c|}{$\begin{array}{l}\text { Percentages are calculated as proportion of non-missing data } \\
\text { Bold text indicates statistical significance for TSRs versus TIRs } \\
O T C \text { over-the-counter, PSC patient self-completion, TIR triptan insufficient responders (based on efficacy), TSR triptan } \\
\text { sufficient responders (based on efficacy) } \\
\text { a Chi-square test } \\
\text { b Fisher's exact test }\end{array}$} \\
\hline
\end{tabular}

(OR 4.244 [95\% CI 1.206, 14.934]; $p=0.024$ ), and for patients with greater migraine severity over the past 3 months (OR 1.202 [95\% CI $1.006,1.435] ; p=0.043)$. Patients with a higher EQ-5D-5L utility score were less likely to be a TIR (OR 0.670 [95\% CI 0.539, 0.833]; $p<0.001)$.

\section{DISCUSSION}

The results of this analysis of real-world data from consulting Japanese patients currently receiving a triptan as their only prescribed acute medication for migraine adds to the sparse level of data currently available on people reporting sufficient/insufficient efficacy from triptan acute treatment in Japan. Previous studies have addressed unmet patient needs in both acute and preventive therapy for episodic and chronic migraine [6], and insufficient response to acute treatment [7], but to our knowledge this is the first analysis to focus specifically on patients who do not respond sufficiently to triptan treatment. Our data show that TIRs have worse migraine-related disability, HRQoL, and work productivity than TSRs. Findings include lower levels of migraine-specific HRQoL across all domains of the MSQ, lower EQ-5D-5L utility 
scores, and greater work and activity impairments in TIRs than in TSRs.

Similar findings were reported in a global analysis of 2017 Adelphi Migraine DSP data from the USA, France, Germany, Italy, Spain, and the UK, which also found lower levels of migraine-specific HRQoL across all domains of the MSQ and lower EQ-5D-5L utility scores in TIRs than in TSRs (defined using the same definitions as in the current survey). However, this global analysis reported that an insufficient response to triptan acute treatment impacted significantly on work productivity across all four components of the WPAI, including work time missed [28]. A lack of difference in work time missed between TIRs and TSRs in the current research may reflect cultural differences between these countries and Japan concerning work ethic and coping with pain and discomfort.

This lower work productivity in TIRs than in TSRs could have financial implications for workers. In 2013, the results of a survey suggested that the presence of migraine or chronic headaches among Japanese workers aged 18-59 years not only resulted in a mean of 0.9 -6.2 days' absence from work over a 4-week period but also impacted performance at work, leading to wage loss in Japanese workers [30]. The effect of migraine or chronic headaches on work days missed or loss of working hours while at work varied by age group. Men and women aged 50-59 years were most affected by work days missed (absenteeism) and by the consequent loss of wages. Lost working hours while at work (presenteeism) and the associated wage loss was greatest among men aged 30-39 years and women aged $40-49$ years. Total wage loss over a 4-week period because of work days missed and work hours lost ranged from USD707 to USD1603 in men and from USD596 to USD944 in women.

In the global analysis of 2017 Adelphi Migraine DSP data, TIRs were significantly more likely than TSRs to experience anxiety, stress, depression, and sleeping disorders [28]. The presence of depression was also identified as related to an inadequate 2 -h pain-free response to acute medication (defined as pain freedom in $<50 \%$ of migraine attacks) in the American
Migraine Prevalence and Prevention real-world study [31]. In contrast, this analysis of the 2017 Japan Adelphi Migraine DSP identified no significant differences in any concomitant comorbidities between TIRs and TSRs. A similar finding was reported in the 2014 Japan Adelphi Migraine DSP analysis, which aimed to identify factors associated with an insufficient response to acute medication (including but not limited to triptans) in people with migraine [8]. These differences between analyses of global and Japan Adelphi Migraine DSP data may reflect differences in the health care systems of Japan and the countries included in the global analysis.

It should be noted that the lower migrainespecific HRQoL seen in TIRs than in TSRs across all domains in the current analysis (difference in MSQ Role Function - Restrictive score: 6.2 points; Role Function - Preventive score: 5.5 points, and Emotional Function score: 8.6 points) was both statistically significant ( $p$ $\leq 0.015$ ) and clinically meaningful (minimal important differences [MIDs]: 3.2, 4.6, and 7.5 points, respectively [32]). In addition, comparison of the EQ-5D-5L utility score for TIRs in the current study (0.80) with reported Japanese norms (mean 0.950-0.899 for age range $>20-29$ to $<70$ years [33]) further suggests that HRQoL is markedly impaired in Japanese people with migraine with insufficient triptan efficacy during acute therapy. The lower headache-related disability seen in TIRs than in TSRs in the current study (difference in MIDAS total score: 7.3) was also clinically meaningful (MID: 3.7 [34]) but not statistically significant in adjusted analyses (difference was both significant and clinically meaningful in non-adjusted analyses).

Freedom from pain $2 \mathrm{~h}$ after taking medication is a recommended endpoint for assessing the efficacy of an acute treatment for migraine [11] and is a factor considered important by patients [35]. Consistency of efficacy is also an important outcome for patients [36]. Previously proposed cut-offs for sufficient response to acute treatments for migraine included pain freedom at $2 \mathrm{~h}$ in at least two of three $(67 \%)$ treated attacks [37] or three of four attacks (75\% [38]), and headache relief within $2 \mathrm{~h}$ in at least 
(a) MSQ Total Score

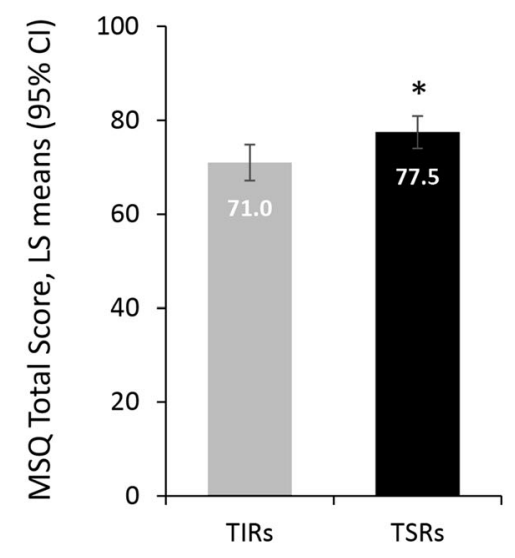

MSQ Domain Score

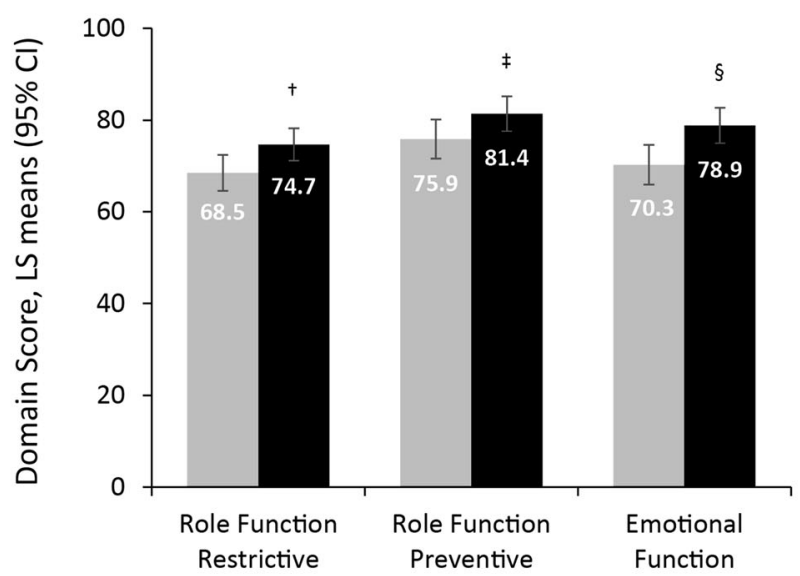

TIRs (b)

EQ-5D Utility Score

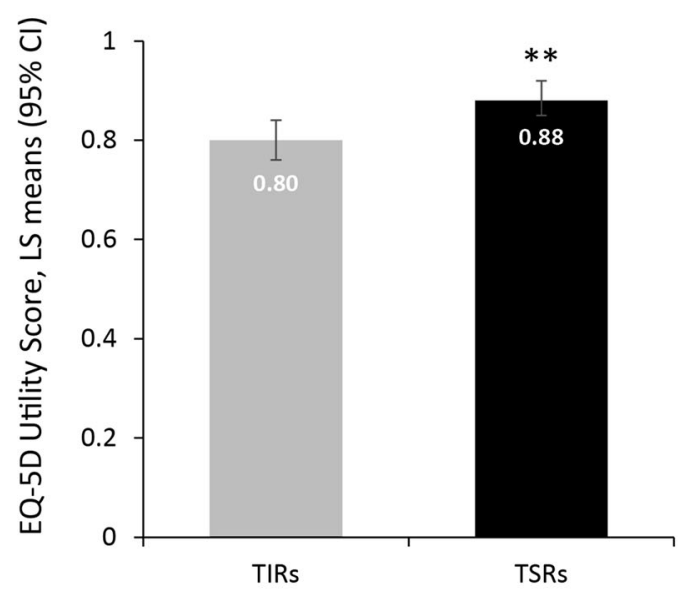

(c)

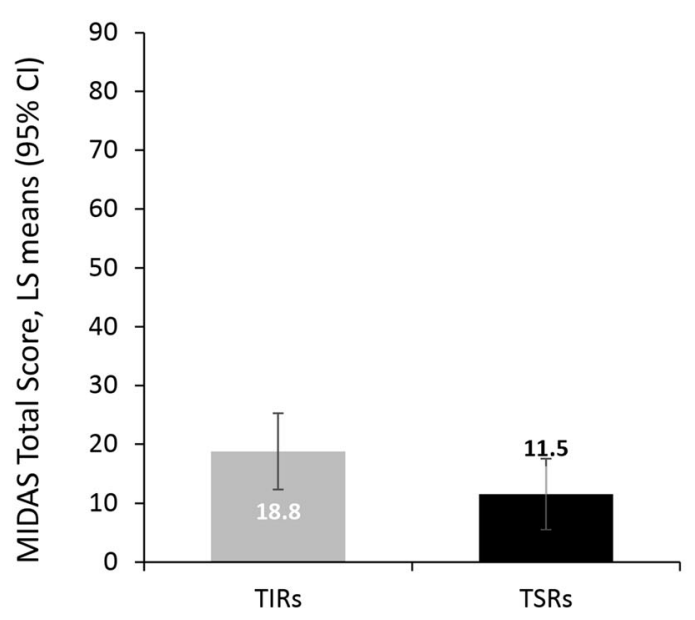

EQ-5D VAS Score

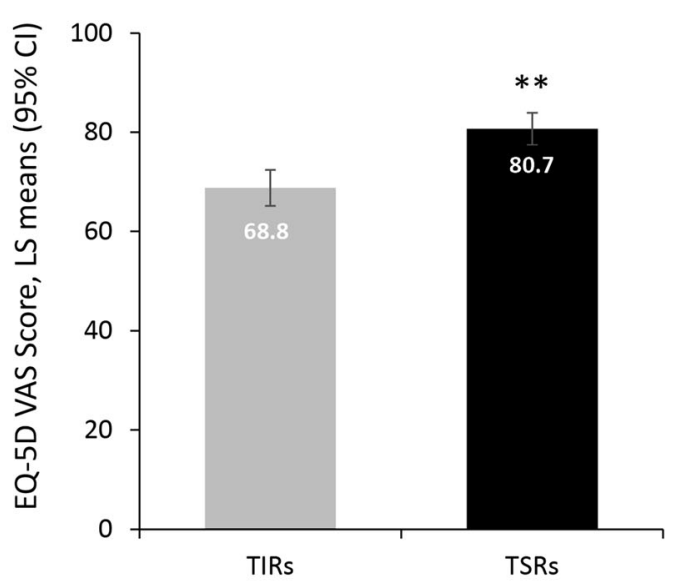


4Fig. 2 Adjusted results in triptan insufficient responders (TIRs) and triptan sufficient responders (TSRs) (response based on efficacy): health-related quality of life: (a) MSQ scores and (b) EQ-5D-5L scores; and (c) disability: MIDAS scores. Data were controlled for age, sex, migraine headache day frequency, comorbidities, preventive medication use, and with or without aura. Higher scores on MIDAS indicate greater disability; higher scores on MSQ and EQ-5D-5L indicate better quality of life and health state, respectively. ${ }^{*} p=0.009,{ }^{\dagger} 0.015,{ }^{\star} 0.043,{ }^{\S} 0.002$, ** $<0.001$ for TSRs versus TIRs. CI confidence interval, $E Q-5 D$ EuroQol 5-dimension questionnaire, EQ-5D-5L EuroQol 5-dimension 5-level questionnaire, $L S$ least squares, MIDAS Migraine Disability Assessment, $M S Q$ Migraine-Specific Quality of Life questionnaire, $V A S$ visual analog scale

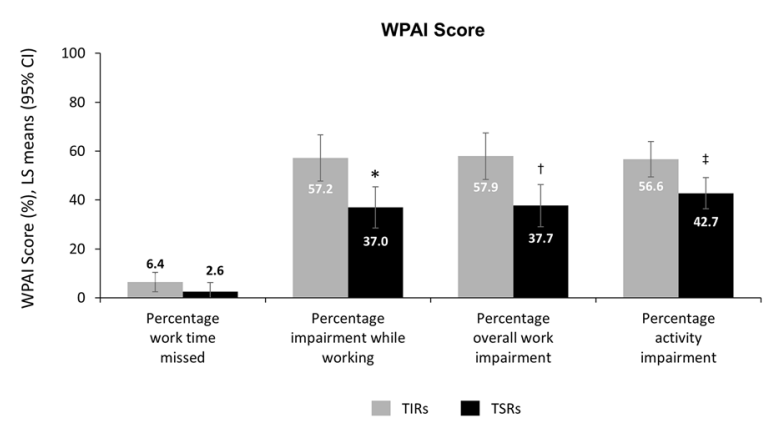

Fig. 3 Adjusted results in triptan insufficient responders (TIRs) and triptan sufficient responders (TSRs) (response based on efficacy): WPAI scores. Data were controlled for age, sex, migraine headache day frequency, comorbidities, preventive medication use, and with or without aura. ${ }^{*} p=0.001,{ }^{\dagger} 0.002,{ }^{\ddagger} 0.003$ for TSRs versus TIRs. CI confidence interval, $L S$ least squares, WPAI Work Productivity and Activity Impairment questionnaire

four of five attacks [39]. We therefore consider that the definition of sufficient triptan efficacy used in our study-freedom from pain $2 \mathrm{~h}$ after taking medication in at least four of five migraine attacks-is appropriate for an acute medication. Using this same definition, the global analysis of 2017 Adelphi Migraine DSP data reported that $34 \%$ of patient respondents were TIRs [28].

The proportions of patients noting an insufficient response to triptan acute therapy reported in the 2017 global Adelphi Migraine DSP (34\%) [28] and in the current Japan Adelphi
Migraine DSP (44\%) are lower than those cited in the above-mentioned studies by Visser et al. [39] (48\%) and Ho et al. [38] (52\%), which used less stringent response criteria. Higher rates of insufficient response to triptan therapy (50\%-78\%) were also found in a network metaanalysis of randomized controlled trials comparing triptans with placebo or active migraine treatments [10]. However, the definition used in this meta-analysis, freedom from pain at $2 \mathrm{~h}$, did not assess consistency of response.

TIRs exhibited significantly more monthly headache days and greater migraine severity over the past 3 months than TSRs, a finding reflected in the 2017 global analysis of Adelphi Migraine DSP data [28]. Visser et al. [39] also reported more severe vomiting and photophobia, more often needing to sleep or rest, and more frequently experiencing initial worsening of the headache in non-responders to oral sumatriptan than in responders. These findings suggest that underlying disease severity, with some disease being difficult to treat, may be a reason for insufficient triptan efficacy when these agents are used as acute treatment.

TIRs were significantly more likely to use OTC and preventive treatments than TSRs, findings also reported in the global analysis of 2017 Adelphi Migraine DSP data [28]. Whereas OTC medication use has been shown to be common among US people with migraine $[40,41]$, a recent article suggested that in Japan, OTC drugs are used mainly for minor health problems and that Japanese patients prefer prescription medications or getting a physician's advice for their health problems [42]. Our results therefore suggest that TIRs have a clear need for OTC medications to relieve a migraine attack that is not being adequately met by prescribed triptans.

Over half $(52.3 \%)$ of TIRs were currently prescribed a preventive medication in the current study, and similar levels of usage (54.2\%) in TIRs were reported in the global analysis of 2017 Adelphi Migraine DSP [28]. These levels of preventive medication use are in line with the finding of greater headache severity in this population, and with Japanese guidelines, which recommend preventive medication for patients in whom disability in daily living due 
Table 5 Logistic regression modeling of insufficient triptan efficacy with backward selection results $(N=200)$

\begin{tabular}{|c|c|c|c|c|}
\hline \multirow[t]{2}{*}{ Factor } & \multicolumn{4}{|l|}{ Odds ratio estimates } \\
\hline & Effect & $\begin{array}{l}\text { Point } \\
\text { estimate }\end{array}$ & $\begin{array}{l}95 \% \\
\text { Wald } \\
\text { CIs }\end{array}$ & $p$ value \\
\hline $\mathrm{Age}^{\mathrm{a}}$ & Older vs. younger & 0.933 & $\begin{array}{l}0.965 \\
1.021\end{array}$ & 0.6114 \\
\hline $\operatorname{Sex}^{\mathrm{a}}$ & Female vs. male & 2.215 & $\begin{array}{l}0.969 \\
5.064\end{array}$ & 0.0595 \\
\hline $\begin{array}{l}\text { When do you usually take your } \\
\text { prescribed acute }\end{array}$ & $\begin{array}{l}\text { "After pain has started and I have an idea of how severe it } \\
\text { is" vs. "At first sign of a migraine (before pain starts)" }\end{array}$ & 4.244 & $\begin{array}{l}1.206 \\
14.934\end{array}$ & 0.0243 \\
\hline medication $^{\mathrm{a}^{\mathrm{a}}}$ & $\begin{array}{l}\text { "When pain starts" vs. "At first sign of a migraine (before } \\
\text { pain starts)" }\end{array}$ & 1.493 & $\begin{array}{l}0.730 \\
3.055\end{array}$ & 0.2722 \\
\hline Physician specialty ${ }^{\mathrm{b}}$ & Neurologist vs. internist & 1.971 & $\begin{array}{l}0.940 \\
4.131\end{array}$ & 0.0724 \\
\hline $\begin{array}{l}\text { Currently taking OTC } \\
\text { medications } s^{\mathrm{a}}\end{array}$ & "Yes" vs. "No" & 4.679 & $\begin{array}{l}1.524 \\
14.371\end{array}$ & 0.0070 \\
\hline $\begin{array}{l}\text { Ever received preventive } \\
\text { medication }{ }^{\mathrm{b}}\end{array}$ & "Yes" vs. "No" & 1.780 & $\begin{array}{l}0.909 \\
3.486\end{array}$ & 0.0928 \\
\hline EQ-5D-5L utility scores ${ }^{\mathrm{a}}$ & Higher vs. lower (range $0-1$ ) & 0.670 & $\begin{array}{l}0.539 \\
0.833\end{array}$ & 0.0003 \\
\hline $\begin{array}{l}\text { Severity of migraine over past } 3 \\
\text { months }{ }^{b}\end{array}$ & Higher vs. lower $^{\mathrm{c}}$ & 1.202 & $\begin{array}{l}1.006 \\
1.435\end{array}$ & 0.0425 \\
\hline
\end{tabular}

The results in the table are from a backward logistic regression with a significance level of 0.1 for a variable to stay in the model. Candidate categorical covariates included physician specialty, patient sex, diagnosis of migraine with aura, diagnosis of migraine without aura, diagnosis of tension-type headache, comorbidities (depression, anxiety, neck pain), diagnosis of menstrual migraine or menstrual-related migraine, symptoms currently experienced (nausea, vomiting), when acute medication is taken relative to the start of the attack ("at the first sign of a migraine" [before the pain starts], "when the pain starts," or "after the pain has started and I have an idea of how severe it is"); taking OTC medication for migraine attacks, and whether the patient ever received preventive medication for their migraine

Bold text indicates statistical significance for triptan sufficient responders versus triptan insufficient responders

$C I$ confidence interval, EQ-5D-5L EuroQol 5-dimension 5-level questionnaire, OTC over-the-counter

${ }^{a}$ Patient-reported

b Physician-reported

${ }^{c}$ Reported using a scale from 1 (very mild) to 10 (very severe)

to migraine is not adequately relieved by acute treatment alone [9]. However, considerably lower levels of preventive medication use have been reported in other real-world studies in patients with migraine (e.g., $11.5 \%$ in the Migraine in America Symptoms and Treatment [MAST] study [40]). One possible reason for this discrepancy could be the fact that in Adelphi Migraine DSP surveys, patients are actively consulting their physician, whereas MAST was a postal-based longitudinal survey of respondents with migraine.

Satisfaction with medication has been shown to be predictive of sustained use of 
triptans for the treatment of migraine [43] and of patients' willingness to continue with their current prescribed acute therapy for migraine [8]. In the current analysis, although a high proportion of patients reported that they were satisfied with their prescribed triptan acute medication, TIRs were more likely than TSRs to express lower levels of satisfaction, or even dissatisfaction, a finding also reported in the global analysis of 2017 Adelphi Migraine DSP data [28].

Logistic regression analysis identified several factors as being significantly associated with insufficient triptan efficacy as acute medication in this study. These included treatment-related issues-the timing of triptan administration (taking the triptan after pain had started rather than at first sign of a migraine attack) and use of OTC medication-as well as greater migraine severity during the past 3 months and lower EQ5D-5L utility scores (indicating lower general health). Of these, only timing of acute medication was identified in the previous analysis of predictors of insufficient response to any acute migraine medication among patients in Japan [8]. Early administration of triptan medication (taking acute prescribed medication when/after pain started rather than at first sign of a migraine attack), use of OTC medication, migraine severity during the past 3 months, and EQ-5D utility scores were also identified as factors associated with response to triptan acute medication in the global analysis of 2017 Adelphi Migraine DSP data, which included a larger patient population [28]. Additional factors identified included the MSQ Role Function - Preventive and Emotional Function domains and headache frequency (higher average number of monthly headache days over the past 3 months).

\section{Strengths/Limitations}

A major strength of this study was the use of real-world data collected using a standardized methodology (as part of the Adelphi Migraine DSP [21]), thus facilitating the comparison of study findings with those from other countries. Further strengths and limitations of the use of
Japan Adelphi Migraine DSP data have previously been reported $[7,8]$. As all patients who participated in the Japan Adelphi Migraine DSP had a physician-confirmed diagnosis of migraine, the study findings can be considered representative of consulting patients with migraine in Japan. As expected, given the realworld nature of the data analyzed, differences were identified between TIRs and TSRs. When investigating differences in outcomes between TIRs and TSRs, these potential confounders were accounted for in adjusted analyses.

The main limitations of this study are that the data were cross-sectional in nature (hence, causality cannot be inferred) and that only a limited number of physicians and patients participated (few patients who participated in the Japan Adelphi Migraine DSP were currently receiving acute treatment with triptan monotherapy for their migraine).

\section{CONCLUSION}

Results from this analysis, demonstrating the negative impact of migraine attacks on the HRQoL, ability to perform in daily life, and work productivity of people with migraine in Japan who report insufficient efficacy with acute treatment with triptans, highlight the need for additional effective acute treatment options.

\section{ACKNOWLEDGMENTS}

Funding. Adelphi Migraine DSP was an independent cross-sectional survey conducted by Adelphi Real World. Eli Lilly and Company purchased access to the dataset from which this analysis and publication are derived and funded the study. Eli Lilly and Company are funding the Rapid Service Fee for publication.

Medical Writing, Editorial and Other Assistance. The authors would like to acknowledge Gill Gummer and Caroline Spencer (Rx Communications, Mold, UK) for medical writing assistance with the preparation of 
this manuscript, funded by Eli Lilly and Company.

Authorship. All named authors meet the International Committee of Medical Journal Editors (ICMJE) criteria for authorship for this article, take responsibility for the integrity of the work as a whole, and have given their approval for this version to be published.

Authorship Contributions. All authors had full access to all of the data in this study and take complete responsibility for the integrity of the data and accuracy of the data analysis. All authors have given their approval for this version to be published in Pain and Therapy.

Disclosures. Koichi Hirata has received grants from the Ministry of Health, Labor and Welfare and the Japan Agency for Medical Research and Development, and honoraria from Alexion Pharmaceuticals, Inc.; Amgen; Esai; Otsuka; Novartis; AbbVie; Pfizer; Novartis; and Eli Lilly outside of this study. Kaname Ueda, Mika Komori, Wenyu Ye, Yongin Kim, and Tamas Treuer are full-time employees and minor stockholders of Eli Lilly and Company. Sarah Cotton and James Jackson are full-time employees of Adelphi Real World.

Compliance with Ethics Guidelines. Patients provided informed consent for use of their anonymized and aggregated data for research and publication in scientific journals by means of a checkbox on the front page of the PSC. Data were collected in such a way that patients and physicians could not be directly identified. All data were aggregated and deidentified before receipt by Adelphi Real World. The DSP methodology and questionnaires were reviewed by an official independent body, FEKI, which granted ethical approval. As this was a retrospective analysis that used deidentified data that had been previously collected, patient participants were not required to provide formal Consent to Release Information forms for the current analyses; the original consent from those who provided data in the DSP covered the planned analyses in this study. The study was conducted in accordance with the applicable regulations of Japan (the Ethical Guidelines for Medical and Health Research Involving Human Subjects).

Data Availability. The data that support the findings of this study are available from Adelphi Real World, but restrictions apply to the availability of these data, which were used under license for the current study and so are not publicly available. However, data are available from the authors upon reasonable request and with permission from Adelphi Real World.

Open Access. This article is licensed under a Creative Commons Attribution-NonCommercial 4.0 International License, which permits any non-commercial use, sharing, adaptation, distribution and reproduction in any medium or format, as long as you give appropriate credit to the original author(s) and the source, provide a link to the Creative Commons licence, and indicate if changes were made. The images or other third party material in this article are included in the article's Creative Commons licence, unless indicated otherwise in a credit line to the material. If material is not included in the article's Creative Commons licence and your intended use is not permitted by statutory regulation or exceeds the permitted use, you will need to obtain permission directly from the copyright holder. To view a copy of this licence, visit http://creativecommons.org/licenses/by$\mathrm{nc} / 4.0 /$.

\section{REFERENCES}

1. GBD 2017 Disease and Injury Incidence and Prevalence Collaborators. Global, regional, and national incidence, prevalence, and years lived with disability for 354 diseases and injuries for 195 countries and territories, 1990-2017: a systematic analysis for the Global Burden of Disease Study 2017. Lancet. 2018;2018(392):1789-858.

2. GBD 2016 Headache Collaborators. Global, regional, and national burden of migraine and tensiontype headache, 1990-2016: a systematic analysis for the Global Burden of Disease Study 2016. Lancet Neurol. 2018;17:954-76. 
3. GBD 2016 Disease and Injury Incidence and Prevalence Collaborators. Global, regional, and national incidence, prevalence, and years lived with disability for 328 diseases and injuries for 195 countries, 1990-2016: a systematic analysis for the Global Burden of Disease Study 2016. Lancet. 2017;390:1211-59.

4. Diener HC, Holle-Lee D, Nägel S, Dresler T, Gaul C, Göbel $\mathrm{H}$, et al. Treatment of migraine attacks and prevention of migraine: guidelines by the German migraine and headache society and the German society of neurology. Clin Trans Neurosci. 2019;3: $1-40$.

5. Lipton RB, Buse DC, Serrano D, Halland S, Reed ML. Examination of unmet treatment needs among persons with episodic migraine: results of the American migraine prevalence and prevention (AMPP) Study. Headache. 2013;53:1300-11.

6. Takeshima T, Wan Q, Zhang Y, et al. Prevalence, burden, and clinical management of migraine in China, Japan, and South Korea: a comprehensive review of the literature. J Headache Pain. 2019;20: 111.

7. Ueda K, Ye W, Lombard L, et al. Real-world treatment patterns and patient-reported outcomes in episodic and chronic migraine in Japan: analysis of data from the Adelphi Migraine Disease Specific Programme. J Headache Pain. 2019;20:68.

8. Hirata $\mathrm{K}$, Ueda $\mathrm{K}$, Ye $\mathrm{W}$, et al. Factors associated with insufficient response to acute treatment of migraine in Japan: analysis of real-world data from the Adelphi Migraine Disease Specific Programme. BMC Neurol. 2020;20(1):274.

9. Japanese Society of Neurology and the Japanese Headache Society. Clinical practice guideline for chronic headache 2013. https://www.neurology-jp. org/guidelinem/ch/index.html. (Accessed Apr 22 2020).

10. Cameron C, Kelly S, Hsieh SC, et al. Triptans in the acute treatment of migraine: a systematic review and network meta-analysis. Headache. 2015;55(Suppl 4):221-35.

11. Diener HC, Tassorelli C, Dodick DW, et al. Guidelines of the International Headache Society for controlled trials of acute treatment of migraine attacks in adults: fourth edition. Cephalalgia. 2019;39:687-710.

12. Gilmore B, Michael M. Treatment of acute migraine headache. Am Fam Physician. 2011;83:271-80.

13. Mathew NT, Landy S, Stark S, et al. Fixed-dose sumatriptan and naproxen in poor responders to triptans with a short half-life. Headache. 2009;49: 971-82.

14. Gallagher RM, Kunkel R. Migraine medication attributes important for patient compliance: concerns about side effects may delay treatment. Headache. 2003;43:36-43.

15. Wells RE, Markowitz SY, Baron EP, et al. Identifying the factors underlying discontinuation of triptans. Headache. 2014;54:278-89.

16. Messali AJ, Yang M, Gillard P, et al. Treatment persistence and switching in triptan users: a systematic literature review. Headache. 2014;54: 1120-30.

17. Lipton RB, Marcus SC, Shewale AR, Dodick DW, Viswanathan HN, Doshi JA. Acute treatment patterns in patients with migraine newly initiating a triptan. Cephalalgia. 2020;40:437-47.

18. Lipton RB, Fanning KM, Serrano D, Reed ML, Cady $\mathrm{R}$, Buse DC. Ineffective acute treatment of episodic migraine is associated with new-onset chronic migraine. Neurology. 2015;84:688-95.

19. Bigal ME, Lipton RB. The epidemiology, burden, and comorbidities of migraine. Neurol Clin. 2009;27:321-34.

20. Buse DC, Rupnow MF, Lipton RB. Assessing and managing all aspects of migraine: migraine attacks, migraine-related functional impairment, common comorbidities, and quality of life. Mayo Clin Proc. 2009;84:422-35.

21. Anderson P, Benford M, Harris N, Karavali M, Piercy J. Real-world physician and patient behaviour across countries: disease-specific programmes - a means to understand. Curr Med Res Opin. 2008;24: 3063-72.

22. Bagley CL, Rendas-Baum R, Maglinte GA, Yang M, Varon SF, Lee J, et al. (2012) Validating migrainespecific quality of life questionnaire v21 in episodic and chronic migraine. Headache. 2012;52:409-21.

23. Herdman M, Gudex C, Lloyd A, et al. Development and preliminary testing of the new five-level version of EQ-5D (EQ-5D-5L). Qual Life Res. 2011;20: 1727-36.

24. Stewart WF, Lipton RB, Kolodner K, Liberman J, Sawyer J. Reliability of the migraine disability assessment score in a population-based sample of headache sufferers. Cephalalgia. 1999;19:107-14.

25. Stewart WF, Lipton RB, Dowson AJ, Sawyer J. Development and testing of the migraine disability assessment (MIDAS) questionnaire to assess headache-related disability. Neurology. 2001;56:20-8. 
26. Reilly MC, Zbrozek AS, Dukes EM. The validity and reproducibility of a work productivity and activity impairment instrument. Pharmacoeconomics. 1993;4:353-65.

27. Iigaya M, Sakai F, Kolodner KB, Lipton RB, Stewart WF. Reliability and validity of the Japanese migraine disability assessment (MIDAS) questionnaire. Headache. 2003;43(4):343-52.

28. Lombard L, Farrar M, Ye W, et al. A global realworld assessment of the impact on health-related quality of life and work productivity of migraine in patients with insufficient versus good response to triptan medication. J Headache Pain. 2020;21(1):41.

29. Lombard L, Wenyu Y, Nichols R, et al. A real-world analysis of patient characteristics, treatment patterns, and level of impairment in patients with migraine who are insufficient responders vs responders to acute treatment. Headache. 2020;60(7):1325-39.

30. Wada K, Arakida M, Watanabe R, Negishi M, Sato J, Tsutsumi A. The economic impact of loss of performance due to absenteeism and presenteeism caused by depressive symptoms and comorbid health conditions among Japanese workers. Ind Health. 2013;51:482-9.

31. Lipton RB, Munjal S, Buse DC, Fanning KM, Bennett A, Reed ML. Predicting inadequate response to acute migraine medication: results from the American migraine prevalence and prevention (AMPP) study. Headache. 2016;56:1635-48.

32. Cole JC, Lin P, Rupnow MF. Minimal important differences in the migraine-specific quality of life questionnaire (MSQ) version. Cephalalgia. 2009;29: 1180-7.

33. Shiroiwa T, Fukuda T, Ikeda S, et al. Japanese population norms for preference-based measures: EQ5D-3L, EQ-5D-5L, and SF-6D. Qual Life Res. 2016;25:707-19.

34. Lipton RB, Desai P, Sapra S, Buse DC, Fanning KM, Reed ML. How much change in headache-related disability is clinically meaningful? Estimating meaningful within person change in MIDAS using data from the AMPP study. Headache. 2017;57: 113-226.

35. Lipton RB, Hamelsky SW, Dayno JM. What do patients with migraine want from acute migraine treatment? Headache. 2002;42(Suppl 1):3-9.

36. Lipton RB, Stewart WF. Acute migraine therapy: do doctors understand what patients with migraine want from therapy? Headache. 1999;39(Suppl 2): S20-6.

37. Viana M, Genazzani AA, Terrazzino S, et al. Triptan nonresponders: do they exist and who are they? Cephalalgia. 2013;33:891-6.

38. Ho TW, Olesen J, Dodick DW, et al. Antimigraine efficacy of telcagepant based on patient's historical triptan response. Headache. 2011;51:64-72.

39. Visser WH, de Vriend RH, Jaspers NH, Ferrari MD. Sumatriptan-nonresponders: a survey in 366 migraine patients. Headache. 1996;36:471-5.

40. Lipton RB, Munjal S, Alam A, et al. Migraine in America Symptoms and Treatment (MAST) study: baseline study methods, treatment patterns, and gender differences. Headache. 2018;58:1408-26.

41. Zhang Y, Dennis JA, Leach MJ, et al. Complementary and alternative medicine use among US adults with headache or migraine: results from the 2012 National Health Interview Survey. Headache. 2017;57:1228-42.

42. Watanabe K. Recent social background and consumer views on over-the-counter drugs and selfmedication. Yakugaku Zasshi. 2020;140(3):423-34.

43. Cady RK, Maizels M, Reeves DL, Levinson DM, Evans JK. Predictors of adherence to triptans: factors of sustained versus lapsed users. Headache. 2009;49:386-434. 